\title{
SAPO-34 framework contraction on adsorption of ammonia: a neutron scattering study
}

\author{
Ian P. Silverwood
}

\section{Published version information}

This is the peer reviewed version of the following article:

Citation: IP Silverwood. "SAPO-34 framework contraction on adsorption of ammonia: a neutron scattering study." ChemPhysChem, vol. 20, no. 13 (2019): 1747-1751.

DOI: $10.1002 / \mathrm{cphc} .201900230$

which has been published in final form at the DOI above. This article may be used for non-commercial purposes in accordance with Wiley-VCH terms and conditions for self-archiving.

Please cite only the published version using the reference above. This is the citation assigned by the publisher at the time of issuing the AAM. Please check the publisher's website for any updates. 


\title{
SAPO-34 Framework Contraction on Adsorption of Ammonia: A Neutron Scattering Study
}

\author{
Ian P. Silverwood ${ }^{\star[a]}$
}

\begin{abstract}
Neutron scattering data was recorded from SAPO34 using the OSIRIS instrument before and after repeated ammonia adsorption at pressures up to 8 bar. Coherent scattering from the zeolite framework provides the neutron powder diffraction pattern and gave evidence for anisotropic contraction on ammonia dosing. Incoherent quasielastic scattering from the hydrogen of the ammonia showed that mobile ammonia was present in the framework. The quasielastic data was fitted to a model where the ammonia was confined within the chabazite cage in the $c$ direction of the crystal lattice, with diffusion solely occurring through the perpendicular 8membered rings. The calculated diffusion constant reached a maximum of $6.3 \times 10^{-8} \mathrm{~m}^{2} \mathrm{~s}^{-1}$ at 5 bar.
\end{abstract}

\section{Introduction}

Zeolites and their related materials have a range of uses from nuclear waste remediation ${ }^{[1]}$ to cat litter $^{[2]}$ and detergents. ${ }^{[3]}$ One major use is catalysis, ${ }^{[4]}$ due to their high surface area, solid acid functionality and the size selectivity conferred by their porous architecture. This is well exploited in the petrochemical industry and it has been said that "every drop of gasoline has seen the inner structure of several zeolites." ${ }^{\text {"[5] }}$ At the other end of an internal combustion engine, it is likely that the emissions will also encounter zeolitic materials in an exhaust catalyst system to control the release of noxious substances to the atmosphere. ${ }^{[6]}$ Amongst the factors which determine a zeolite's action are the way it concentrates species from the environment and how the adsorbates move and react within the pore network. Every method of measuring diffusion within a pore network has its limitation, and it must be remembered that very different length scales can play a part in how molecules access parts of the structure which otherwise appear identical. Great care is needed to separate the contributions from factors such as reactor packing, particle size and macro-, meso- and micro-porosity.

One technique gaining in importance is quasielastic neutron scattering or QENS. ${ }^{[7]}$ This measures the change in energy and momentum of neutrons scattered from moving atoms, providing a measure of both the magnitude and geometry of motion in the sample. The nature of the technique limits the information accessed to interactions occurring on the molecular scale. As such it provides a measure of what would occur in a perfect bulk zeolite, and effects due to particle size and bulk materials transfer can be neglected. This scale is also well matched to the range of systems that can be accessed by molecular dynamics simulations, and provides a good test of computational theory. ${ }^{[8]}$

\footnotetext{
[a] Dr I.P. Silverwood

ISIS Neutron and Muon Facility

STFC

Rutherford Appleton Laboratory, Harwell Science and Innovation Campus, Oxon OX11 0QX, UK

E-mail: ian.silverwood@stfc.acuk
}

The catalytic selectivity of zeolites is often attributed to the geometric restrictions that pores represent to different molecular sizes. Whilst this intuitively makes sense, a zeolite does not have a single rigid pore size, and these materials often display unpredictable expansion and contraction behaviour. ${ }^{[9]}$ This may present as a negative thermal expansion across a limited temperature range ${ }^{[10]}$ or initial contraction, followed by expansion upon adsorption. ${ }^{[1]}$ The dynamic nature of the pore size is often not addressed, although it seems likely that there will be some degree of framework motion associated with accommodating a guest sorbate molecule. Recently molecular dynamics simulations have shown improved correlation with experiment where flexible model frameworks are used to replace rigid ones $^{[12]}$ and the study of flexibility in zeolite frameworks is increasing. ${ }^{[10,13]}$

The characterisation of heterogeneous catalysts with neutrons is well established. ${ }^{[14]}$ In contrast to $x$-rays which scatter from the electron cloud, scattering of a neutron in a non-magnetic system is from the nucleus of the atom. The strength of the interaction between a neutron and nucleus varies in an apparently random manner, ${ }^{[15]}$ unlike the variation with atomic mass for $x$-rays. This can be considered in terms of (total) neutron cross section, which expresses the likelihood of a scattering event in terms of the apparent area that the particle presents to the neutron. Neutron scattering can also be extremely sensitive to isotopic substitution which is often exploited in both elastic and inelastic scattering techniques. Elastic scattering results in no change in neutron energy and provides information on 'where atoms are'. Inelastic scattering results when the neutron exchanges energy with the atom and tells us 'what atoms do'. ${ }^{[16]}$ Quasielastic neutron scattering measures very small energy changes which can be imagined as elastic collisions from moving particles. This results in a broadening of the elastic peak that can be related to motions within the sample. How the broadening varies with angle in real space (or $Q$ in inverse space) depends on both the rate and the geometry of motion. ${ }^{[17]}$

In addition to this dichotomy of elastic and inelastic scattering, there can be a further subdivision into coherent and incoherent scattering. As neutrons and nuclei are quantum particles, their interaction can be interpreted either as waves or particles. Coherent scattering has the neutron wave scattering in phase from many atoms in the sample simultaneously. Elastically scattered waves interfere and provide the diffraction pattern. Incoherent scattering results from out of phase scattering and provides information about the interaction of the neutron (wave) with a single particle. The total neutron scattering cross section is a sum of the coherent and incoherent scattering cross sections, which vary independently.

Hydrogen has cross sections that vary strongly with isotope. ${ }^{1} \mathrm{H}$ has the highest proportional incoherent cross section, whereas 
the scattering from ${ }^{2} \mathrm{H}$ is dominated by coherent scattering. This effect is heavily exploited in neutron scattering in many ways. For a diffraction experiment, if we want to locate the position of hydrogen atoms it is best to use a deuterated sample to increase coherent scattering. For uncorrelated atomic motions, such as diffusion, a protiated sample is the better probe. ${ }^{[18]}$ For almost all heterogeneous catalytic system, we are interested in the interaction of organic reactants, products and intermediates with an inorganic metal/metal oxide catalyst. In these systems it is commonly accepted as a first approximation that the incoherent neutron scattering can be solely attributed to hydrogen and thus the incoherent scattering represents only the protiated organic species. ${ }^{[19]}$ This allows selective spectroscopic measurements to be made with neutrons in cases where other techniques, such as NMR or optical spectroscopy are incompatible with the inorganic catalyst. It is also possible to obtain simultaneous information regarding the dynamics of the sorbate and the structure of the sorbent from the separate incoherent and coherent scattering.

\section{Results and Discussion}

The chabazite structure consists of a rhombic unit cell with double 6-rings at each vertex with opening $2.7 \AA$. These rings cap cages of $9.4 \AA$ along the c-axis which are linked across the diameter by 8 ring windows of $3.8 \AA$ diameter. ${ }^{[20]}$ The windows define the size of object that may penetrate the interior of the zeolite and define its pore size. Bulk diffusion along the $\mathrm{c}$ axis is possible due to the staggered arrangement of the chabazite cages. However it is not possible for a molecule to travel further than $9.4 \AA$ along the c direction without changing the cage which it occupies.

The neutron diffraction pattern obtained from the sample at different ammonia pressures is displayed in Fig. 1, with expanded sections in Fig. 2. The baseline increases with ammonia pressure due to incoherent scattering from the hydrogen atoms, with no new peaks appearing, which signifies there is little change in the nature of the SAPO-34 structure and the adsorbate is not forming an ordered phase. However, for some features from the framework (see Fig. 2) the peak position shifts markedly. This is most significant for the strongest peak at $9.33 \AA$, which shifts to $9.26 \AA$ on ammonia adsorption. The other features in Fig. 1 show smaller shifts, but all are to lower $d$ spacing demonstrating that the zeolite is contracting on gas adsorption. These shifts are tabulated in Table 1 along with the calculated strain.

The diffraction data indicates anisotropic strain within the zeolite framework, and deformation within the unit cell can be imagined if the effect is strongest at the pore walls. This would result in in a localised strain analogous with surface reconstruction on adsorption. The range of strains displayed in Table 1 supports this conjecture, but higher resolution diffraction data would be needed to carry out Reitveld refinement and was therefore not attempted.

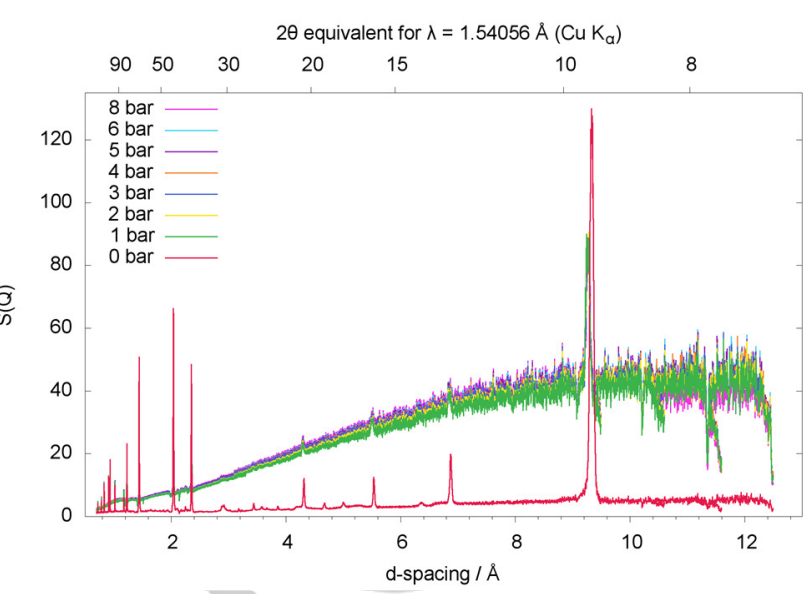

Figure 1. Diffraction patterns for SAPO-34 with different $\mathrm{NH}_{3}$ pressure

Fitting of the incoherent spectroscopic scattering data was carried out using the DAVE software package to convolute the experimentally measured resolution function with a delta function to represent the elastic scattering from static atoms and a single Lorentzian to represent the quasielastic broadening from those moving within the dynamic window of the instrument. A linear background function was used to account for motions that were too fast for the time-window of OSIRIS. An example fit for a single pressure and $Q$. is displayed in Fig. 3, with the variation in the extracted peak breadth at full width, half maximum (FWHM) against $Q^{2}$ and pressure plotted for the full data range in Fig. 4. Various models were assessed to obtain the best fit for the experimental data. The lines shown in Fig. 4 assume Singwi-Sjölander jump diffusion ${ }^{[21]}$ within a confined volume, ${ }^{[22]}$ where the discontinuity coincides with the $9.3 \AA$ diameter of the chabazite cage. The fitting parameters are shown in Table 2.

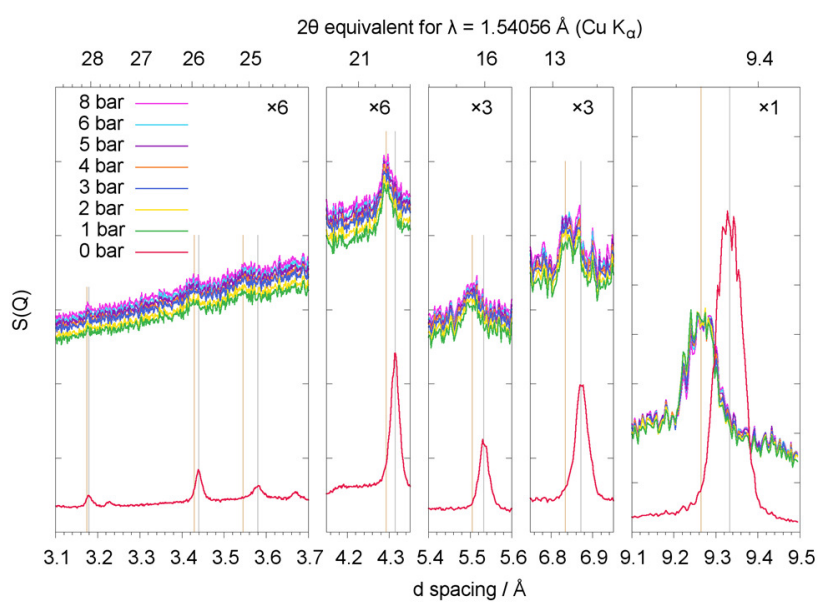

Figure 2. Details of diffraction patterns for SAPO-34 with different $\mathrm{NH}_{3}$ pressure 
The diffusion of ammonia in SAPO-34 has not previously been investigated, although the isostructural analogue chabazite has been of interest due to the selective catalytic reduction of ammonia that is utilised in the treatment of diesel exhaust. Two papers by O'Malley et al. contrasted the diffusion of ammonia in chabazite with levynite ${ }^{[23]}$ and $\mathrm{H}$-chabazite with Cu-chabazite. ${ }^{[24]}$ Self diffusion constants were found to be $7.6 \times 10^{-10}$ and $7.1 \times$ $10^{-10} \mathrm{~m}^{2} \mathrm{~s}^{-1}$ for the $\mathrm{H}$ and $\mathrm{Cu}$ forms respectively, although the data was recorded with lower ammonia loading and is not directly comparable.

Table 1. Peak shifts for SAPO-34 on adsorption of ammonia by neutron powder diffraction (data shown in Fig. 2 .

\begin{tabular}{lllll}
\hline Peak & $\mathrm{d}_{\mathrm{vac}}{ }^{[\mathrm{a}]}$ & $\mathrm{d}_{\mathrm{ads}}{ }^{[\mathrm{a}]}$ & $\Delta \mathrm{d}^{[\mathrm{a}]}$ & Strain $^{[\mathrm{d}]}$ \\
\hline$(1,0,1)$ & 9.33 & 9.265 & -0.068 & -0.00734 \\
$(2,-1,0)$ & 6.872 & 6.835 & -0.037 & -0.00541 \\
$(2,0,-1)$ & 5.533 & 5.505 & -0.028 & -0.00509 \\
$(3,-1,1)$ & 4.315 & 4.293 & -0.022 & -0.00512 \\
$(1,0,4)$ & 3.580 & 3.545 & -0.035 & -0.00987 \\
$(4,-2,0)$ & 3.440 & 3.430 & -0.010 & -0.00292 \\
$(4,1,1)$ & 3.175 & 3.170 & -0.005 & -0.00157 \\
\hline
\end{tabular}

[a] $\ddot{A}[\mathrm{~b}]$ calculated as $\Delta \mathrm{d} / \mathrm{d}_{\mathrm{vac}}$

These works further showed that ammonia did not traverse the 6-ring windows, but was free to move through the 8-ring. In these publications, the motion was fitted to the Chudley-Elliot model, which assumes jumps between lattice points of uniform length. The Singwi Sjölander model better represents the reality of this system as it asserts that there is disordered motion on the sites between jumps, leading to a distribution of jump lengths defined as:

$$
\rho(r)=\frac{r}{r_{0}^{2}} \exp \left(-\frac{r}{r_{0}}\right) \quad 1
$$

If it is assumed that the jump time is negligible compared to the time resident on a site, the broadening is represented by:

$$
\Delta \omega(Q)=\frac{1}{6 \tau} \cdot \frac{Q^{2}\left\langle r^{2}\right\rangle}{1+Q^{2}\left\langle r^{2}\right\rangle / 6}
$$

2

Where $\left\langle r^{2}\right\rangle$ is the mean-squared jump length, and $\mathrm{t}$ is the residence time between jumps. $\Delta \omega$ is the HWHM of the fitted Lorentzian peak, with the diffusion coefficient defined by:

$$
D_{s}=\left\langle r^{2}\right\rangle / 6 \tau
$$

For isotropic diffusion within a non-oriented sample, this model predicts that the broadening approximates $D Q^{2}$ dependence at low $Q$. In a system exhibiting confinement, below the $Q$ value relating to the dimension of confinement, broadening will be constant. In Figure 4, the broadening below $0.46 \AA^{-2}$ is fitted to a constant. This value is chosen as it corresponds to the length of the $\mathrm{CHA}$ cage, $d=9.3 \AA$, using $q=2 \pi / d$. Broadenings above this value were fitted to the Singwi Sjölander model. The model represents confinement inside the $9.3 \AA$ length of the $\mathrm{CHA}$ cage along the c-axis, with free motion perpendicular. This reflects the previous findings that suggest ammonia diffusion cannot occur through the double- 6 window in the chabazite structure. The Lorentzian parameters obtained from DAVE were fitted as a function of $Q^{2}$ using the non-linear least-squares method in gnuplot.

The collected data show the adsorption of ammonia into the chabazite cage, resulting in shrinkage of the zeolite lattice. The literature suggests that both swelling and shrinking of a zeolite may occur when gas is adsorbed, depending on the conditions and interaction between framework and sorbate. ${ }^{[11]}$ The systems reported to date are mostly limited to weakly adsorbed phases that display swelling at moderately elevated pressures.

Exceptions are the work by Wang et al. ${ }^{[25]}$ showing contraction of the framework on $\mathrm{NO}_{2}$ adsorption and contraction induced at extremely high pressures reported by Gatta et al. ${ }^{[26]}$ It should be noted that as zeolites are highly hygroscopic, without appropriate pre-treatment the framework will contain water, and possibly other species captured from the environment. A surface is an energetically disfavoured system and emptying a zeolite entirely requires considerable energy. Accommodation of different guest molecules within the host is expected to result in different geometric change, so it is important to begin from a known, preferably empty state. The strongly adsorbing ammonia appears to only cause contraction in SAPO 34, at least over the pressure range investigated here. Future research into the mechanisms of zeolite distortion when interacting with sorbates may provide a rich field of study towards functional materials. There are already reports of using the expansion effect to improve the performance of membranes for gas separation ${ }^{[27]}$ and other applications of tuning pore size can be easily imagined. 


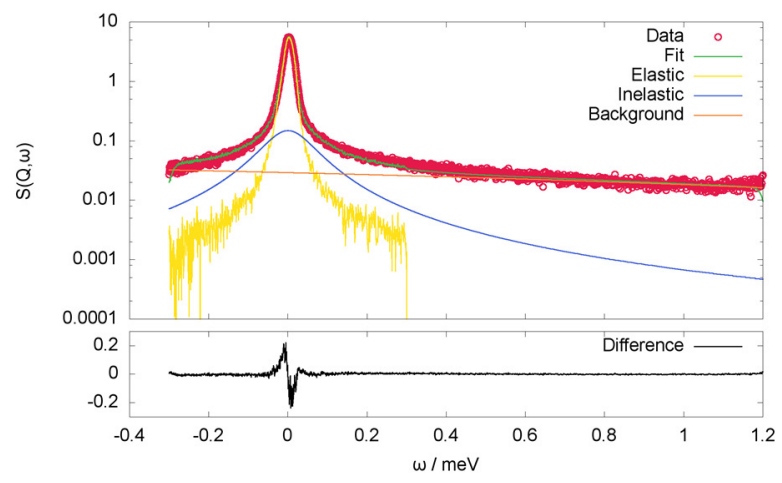

Figure 3. Example fit of Delta-Lorentzian-Background for SAPO34 loaded with ammonia at $325 \mathrm{~K}$

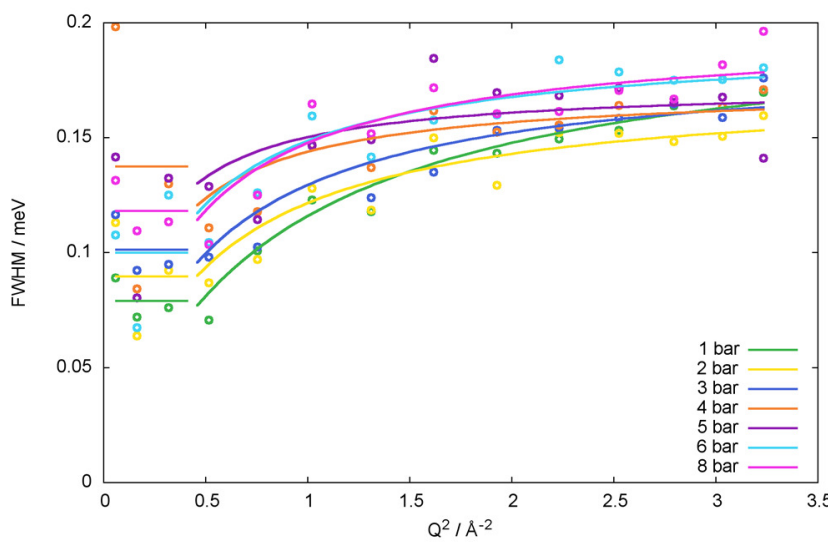

Figure 4. Variation in Lorentzian peak width as a function of $Q$ and ammonia pressure at $325 \mathrm{~K}$. Fits to data below $0.46 \AA^{-2}$ reflect ammonia confinement in the $9.3 \AA$ chabazite cage. Fits above $0.46 \AA^{-2}$ are to the Singwi-Sjölander jump diffusion model representing motion in the perpendicular directions.

The QENS data show an increase in diffusion rate up to 5 bar pressure. Gaseous diffusion will generally increase with pressure, but the confined condensed phase is more similar in density to the liquid phase, ${ }^{[28]}$ although the structure it adopts is more complex. ${ }^{[29]}$ The drop in diffusion above 5 bar is tentatively assigned to steric crowding as more ammonia molecules pack into the framework and decrease the amount of free space for the $\mathrm{NH}_{3}$ to jump into. The factors influencing this maximum are unclear and deserve further study. A quasielastic neutron spectrometer has a limited 'time window' or dynamic range over which processes can be resolved. Processes that are occurring on a longer or shorter time scale will appear as either indistinguishable from the elastic scattering or as a broad baseline that corresponds to a broadening too wide to be quantified. The observable time scale on OSIRIS corresponds well to diffusion, but the data also required a background for acceptable fitting, indicating an additional process was occurring more rapidly. This is likely to represent the rapid rotation of the ammonia. Other possible species present such as the ammonium ion or ammonia co-ordinated to Lewis acid sites are not thought likely to be present in sufficient quantity to affect this interpretation.

Table 2. Diffusion parameters for Singwi-Sjölander model in Fig. 4

\begin{tabular}{llll}
\hline $\mathrm{P}^{[\mathrm{a}]}$ & $\mathrm{T}^{[\mathrm{b}]}$ & $\mathrm{V}\left\langle\mathrm{r}^{2}\right\rangle^{[\mathrm{C}]}$ & $\mathrm{D}_{\mathrm{s}}^{[\mathrm{d}]}$ \\
\hline 1 & 0.619 & 2.81 & $3.54 \times 10^{-9}$ \\
2 & 0.527 & 3.74 & $5.33 \times 10^{-9}$ \\
3 & 0.562 & 3.74 & $5.67 \times 10^{-9}$ \\
4 & 0.523 & 5.53 & $11.52 \times 10^{-9}$ \\
5 & 0.526 & 6.28 & $14.97 \times 10^{-9}$ \\
6 & 0.584 & 4.51 & $8.58 \times 10^{-9}$ \\
8 & 0.597 & 4.25 & $7.80 \times 10^{-9}$ \\
\hline & & \\
\hline [a] Pressure / Bar [b] Time constant / ps [c] Root mean \\
squared jump distance / A [d] Self diffusion coefficient / $\mathrm{m}^{2} \mathrm{~s}^{-1}$
\end{tabular}

For a complete QENS analysis of a mobile organic phase adsorbed on an inorganic crystalline phase it is necessary to distinguish the coherent and incoherent scattering contributions. This is generally approximated as the sum of the elastic coherent, incoherent inelastic and incoherent elastic scatter. It is assumed that the coherent scattering is from the framework alone and coherent inelastic scatter is negligible. The incoherent signal from the mobile phase is calculated by subtracting the Bragg scattering signal of the empty framework from the data recorded with the mobile adsorbate/adsorbent system. The shifting of the Bragg peaks on adsorption shown here call this methodology into doubt, as the elastic signal from the unloaded zeolite is visibly different from the ammonia-occupied system. In many cases this may not cause any issue for the data interpretation, as low Q-resolution will cover such minor shifts in d-spacing. However, it is conceivable that where the Bragg peak lies at the edge of a detector, it may shift to the adjacent one on adsorption, leading to radically different elastic intensities. This should be borne in mind when subtraction is carried out and alternative methods explored. For example it may be better to fit the data from the empty framework with a delta peak, and subtract the height of this peak from the fitted delta peak in the loaded sample. This will account for shifts in peak position, although not in intensity. This may be of concern as the neutron scattering length density from a filled and an empty pore are 
different, ${ }^{[30]}$ but the magnitude of this effect is less likely to create artefacts

\section{Conclusions}

Adsorption of ammonia onto SAPO-34 results in an anisotropic contraction of the zeolite framework at gas pressures between 1 and 8 bar. A mobile condensed ammonia phase is present in the framework where the extent of observed diffusion in the $c$ direction is limited by the length of the CHA cage in the zeolite. Perpendicular diffusion occurs through the 8-ring window. This contrasts with the previously observed framework expansions seen on physisorption carried out with less reactive gases. This framework flexibility is of interest in its own right but also has implications for the interpretation of QENS data obtained from sorbed phases in zeolites.

\section{Experimental Section}

SAPO-34 was obtained from ACS Material with a Si:Al ratio of 0.5 , and activated by heating to $723 \mathrm{~K}$ in a sealed vessel attached to a turbomolecular pump overnight. This was then transferred to an aluminium sample holder of annular geometry in an inert gas glovebox. The sample can was attached to gas handling apparatus that allowed remote dosing of gas whilst the sample was held in the neutron beam. Neutron scattering measurements used the OSIRIS ${ }^{[31]}$ instrument at the ISIS neutron and muon source in Oxfordshire, UK. This instrument can be run as a high energy resolution spectrometer or as a diffractometer. The evacuated sample was measured at $325 \mathrm{~K}$ in both QENS and diffraction modes, before ammonia (CK gas, $99.98 \%$ ) was admitted, and repeated measurements taken at 1, 2, 3, 4, 5, 6 and 8 bar.

QENS spectra used the offset (002) reflection of the pyrolitic graphite analyser bank. This gives an energy transfer range of -0.3 to $1.2 \mathrm{meV}$ with an energy resolution of $24.5 \mu \mathrm{eV}$. OSIRIS has $42{ }^{3} \mathrm{He}$ spectroscopy detectors viewing the graphite analyser that cover a $2 \theta$ range of $11-148$ degrees. In the PG002 configuration, this corresponds to $0.442-1.854 \AA$ ${ }^{1}$. It is common to group these detectors to improve the signal to noise ratio at the expense of $Q$ resolution. The choice of groups was optimised for quasielastic signal, with three detectors summed to provide 14 data points in $Q$.

\section{Acknowledgements}

Experiments at the ISIS Neutron and Muon Source were supported by a beamtime allocation RB1810824 from the Science and Technology Facilities Council. The raw data for this study are available from DOI: $10.5286 /$ ISIS.E.90681395. [32] Prof. Andrew Beale and Dr Ines Lezcano Gonzalez of UCL are thanked for useful discussions on the nature of zeolites and the kind gift of SAPO-34 used in this study.

Keywords: Zeolites • Adsorption • Neutron Diffraction • Diffusion
Owens, M. Higgins-Bos, K. Smith, L. O’Brien, N. Smith, N. Bryan. J. Radioanal. Nucl. Chem. $20183182473-2491$.

[2] A. M. Kiliç, Özen Kiliç, Asian J. Chem. 200618 1405-1412.

[3] S. U. Meshram, U. R. Khandekar, S. M. Mane, A. Mohan, J Surfactants Deterg. 2015 18259-266.

[4] J. Weitkamp, Solid State Ion. 2000 131175-188.

[5] V. Van Speybroeck, K. Hemelsoet, L. Joos, M. Waroquier, R. G. Bell, C. Richard A. Catlow, Chem. Soc. Rev. 201544 7044-7111.

[6] S. Dahlina, C. Lanttob, J. Englund, B. Westerberg, F. Regali, M. Skoglundh, L. J. Pettersson, Catal. Today 2019 320 72-83.

[7] M. Bée. Quasielastic Neutron Scattering. Adam Hilger, Bristol,

1998.

[8] G. Goret, B. Aoun, E. Pellegrini, J. Chem. Inf. Model. 2017 571-5.

[9] B. Ilić, S. G. Wettstein, Micropor. Mesopor. Mater. 2017239221

234.

[10] S. G. Sorenson, J. R. Smyth, R. D. Noble, J. L. Falconer. Ind. Eng Chem. Res. 200948 10021-10024.

[11] G. Y. Gor, P. Huber, N. Bernstein, Appl. Phys. Rev. 20174 011303.

[12] A. J. OMalley, C. R. A. Catlow, Phys. Chem. Chem. Phys. 201315 19024-19030.

[13] A. Sartbaeva, S. A. Wells, M. M. J. Treacy, M. F. Thorpe, Nat. Mater. 20065 962-965.

[14] E. Gibson, I. P. Silverwood in Modern Developments in Catalysis (Eds.: G. J. Hutchings, M. G. Davidson, C. R. A. Catlow, C. Hardacre, N. J. Turner, P. Collier), World Scientific, London 2016 pp. 41-88.

[15] V. F. Sears, Neutron News 19923 26-37.

[16] NobelPrize.org (1994) The Nobel Prize in Physics 1994 [Press Release] <https://www.nobelprize.org/prizes/physics/1994/press-release/>

[17] H. Jobic, D. N. Theodorou, Micropor. Mesopor. Mater. 2007102

21-50.

[18] D. S. Sivia. Elementary Scattering Theory For X-ray and Neutron Users. Oxford University Press, Oxford 2011.

[19] P. C. H. Mitchell, S. F. Parker, A. J. Ramirez-Cuesta, J.

Tomkinson. Vibrational Spectroscopy with Neutrons. World Scientific, London 2005.

[20] Q. Sun, Z. Xie, J. Yu, Nat. Sci. Rev., 2018, 5, 542

[21] K. S. Singwi, A. Sjölander, Phys. Rev. 1960119 863-871.

[22] F. Volino, J-C. Perrin, S. Lyonnard, J. Phys. Chem. B 2006110 $11217-11223$

[23] A. J. O'Malley, M. Sarwar, J. Armstrong, C. R. A. Catlow, I. P. Silverwood, A. P. E. York and I. Hitchcock Phys.Chem.Chem.Phys., 2018, 20, 11976

[24] A. J. O'Malley, I. Hitchcock, M. Sarwar, I. P. Silverwood, S.

Hindocha, C. R. A. Catlow, A. P. E. York, P. J. Collier

Phys.Chem.Chem.Phys., 2016, 18, 17159

[25] X. Wang, J. C. Hanson, J. Szanyi, J. A. Rodriguez, J. Phys. Chem B $200410816613-16616$.

[26] G. D. Gatta, Y. Lee, Mineral. Magazine 2014 78, 267-291.

[27] M. Yu, R. D. Noble, J. L. Falconer, Acc. Chem. Res. 201144 , 1196-1206.

[28] I. P. Silverwood, V. Garcí Sakai, Chem. Eng. Sci. 2018186116

121.

[29] M. Falkowska, D. T. Bowron, H. Manyar, T. G. A. Youngs, C. Hardacre, Angew. Chem. Int. Ed. 2018 57 4565-4570

[30] T. G. A. Youngs, H. Manyar, D. T. Bowron, L. F. Gladden, C Hardacre, Chem. Sci. 2013, 4 3484-3489.

[31] a) M. T. F Telling, K. H. Andersen, Phys. Chem. Chem. Phys. 2004

71255-1261; b) M. T. F. Telling, S. I. Campbell, D. Engberg, D. Martin y

Marero, K. H. Andersen Phys. Chem. Chem. Phys. 2016188423.

[32] I. P. Silverwood The Effects of Zeolite Expansion on Molecular Diffusion. STFC Data Journal 2018 doi:10.5286/ISIS.E.96377625. 
Entry for the Table of Contents (Please choose one layout)

Layout 1:

ARTICLE

Zeolites show interesting deformation characteristics when gas molecules condense in their framework. The strong interaction with ammonia causes the unit cell to shrink, in contrast to the previously seen expansion with weakly adsorbed species.

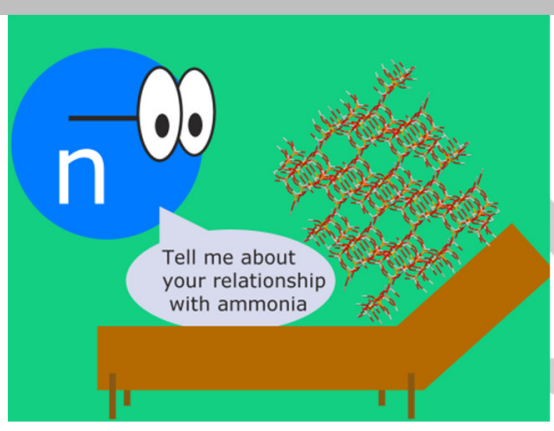

I.P. Silverwood*

Page No. - Page No.

SAPO-34 Framework Contraction on Adsorption of Ammonia: A Neutron Scattering Study

Layout 2:

ARTICLE

Tovt for Tahla of Contante

Author(s), Corresponding Author(s)*

((Insert TOC Graphic here))

Page No. - Page No.

Title 